\title{
MORTALIDADE DE PESSOAS IDOSAS EM DECORRÊNCIA DE EPISÓDIOS DE QUEDAS EM MINAS GERAIS
}

João Marcos Carvalho Oliveira Castro, José Sergio Gama Batista Filho, Francely de Castro e Sousa, Emília Pio da Silva. Mortalidade de pessoas idosas em decorrência de episódios de quedas em Minas Gerais. Revista Saúde Dinâmica, vol. 2, núm.4, 2020. Faculdade Dinâmica do Vale do Piranga.

\section{SAÚDE DINÂMICA - Revista Científica Eletrônica FACULDADE DINÂMICA DO VALE DO PIRANGA}

6a Edição 2020 | Ano II - no 4 | ISSN-2675-133X 


\title{
Mortalidade de pessoas idosas em decorrência de episódios de quedas em Minas Gerais
}

\author{
Mortality of elderly people due to falls in the state of Minas Gerais
}

\author{
João Marcos Carvalho Oliveira Castro ${ }^{1}$, José Sergio Gama Batista Filho ${ }^{2}$, Francely de Castro e \\ Sousa ${ }^{3}$ Emília Pio da Silva ${ }^{4}$ \\ ${ }^{I}$ Discente de Fisioterapia, Faculdade Dinâmica do Vale do Piranga, Ponte Nova, Brasil, ORCID: 0000-0003-0963-0589 \\ ${ }^{2}$ Discente de Fisioterapia, Faculdade Dinâmica do Vale do Piranga, Ponte Nova, Brasil, ORCID:0000-0020-5443-4280 \\ ${ }^{3}$ Docente de Fisioterapia, Faculdade Dinâmica do Vale do Piranga, Ponte Nova, Brasil, ORCID:0000-0002-1881-6409 \\ ${ }^{4}$ Docente de Fisioterapia, Faculdade Dinâmica do Vale do Piranga, Ponte Nova, Brasil, ORCID:0000-0001-8130-5196 \\ Autor correspondente: joaomcarvalhoo@outlook.com
}

\section{Resumo}

O processo de envelhecimento é acompanhado por alterações físicas, biológicas e cognitivas que provocam a redução da capacidade funcional do organismo humano. Tais alterações predispõe a ocorrência de quedas que está entre as principais causas externas de mortalidade em pessoas idosas no Brasil, se tornando um problema de saúde pública. Objetivou-se descrever a mortalidade de idosos em decorrência de episódios de quedas, em Minas Gerais no período entre 2008 e 2018, especificamente buscou-se evidenciar como a fisioterapia pode ajudar a minimizar a ocorrência deste evento. Trata-se de uma pesquisa descritiva, quantitativa, quanto ao período de segmento, tem-se um estudo longitudinal retrospectivo. Foram utilizados dados secundários do Departamento de Informática do SUS (DATASUS). No período estudado, ocorreram 10.038 óbitos por quedas em idosos. O ano de 2018 apresentou índice de mortalidade $155,27 \%$ maior que o ano de 2008. A mortalidade por quedas na população idosa vem aumentando a cada ano no estado de Minas Gerais, fazendo-se necessárias ações preventivas, educativas e reabilitadoras, como a abordagem da fisioterapia, para reduzir os óbitos por essa causa e as demais intercorrências.

Palavras-chave: Idoso; Quedas; Mortalidade; Fisioterapia.

\begin{abstract}
The aging process is accompanied by physical, biological and cognitive changes that reduce the functional capacity of the human organism. Such changes predispose the occurrence of falls, which is among the main external causes of mortality in elderly people in Brazil, becoming a public health problem. The objective was to describe the mortality of the elderly due to episodes of falls in Minas Gerais in the period between 2008 and 2018, specifically seeking to show how physical therapy can help to minimize the occurrence of this event. This is a descriptive, quantitative research, regarding the segment period, and there is a longitudinal retrospective study. Secondary data from the SUS Computer Department (DATASUS) were used. During the study period, 10,038 deaths due to falls occurred in the elderly. The year 2018 presented a mortality rate $155.27 \%$ higher than the year 2008 . Mortality from falls in the elderly population has been increasing each year in the state of Minas Gerais, making preventive, educational and rehabilitative actions necessary, such as physiotherapy approach, to reduce deaths from this cause and other complications.
\end{abstract}

Key words: Elderly; Falls; Mortality; Physiotherapy. 


\section{INTRODUÇÃO}

A transição demográfica no Brasil está provocando mudanças na pirâmide etária, e a população vem se tornando mais envelhecida, este processo é resultante do aumento da expectativa de vida e da redução das taxas de fecundidade e mortalidade. No ano de 2010, a população idosa atingiu 19,6 milhões, estima-se para o ano de 2030 cerca de 41,5 milhões idosos, e 75,5 milhões em 2060 (IBGE, 2015).

Neste cenário envelhecido destaca-se o Estado de Minas Gerais, visto que em 2010 as pessoas idosas representavam $8,10 \%$ da população, as projeções indicam que em 2060 os idosos atingirão 28,75\% da população total, e Minas se tornará o estado com maior números de idosos no Brasil, superando a média nacional que atingirá 25,49\% (IBGE, 2018).

O processo de envelhecimento é acompanhado por alterações progressivas em diversos sistemas do corpo humano, diminuindo a capacidade funcional e tornando o idoso susceptível a quedas (ANDRADE et al., 2017). O comprometimento do sistema motor e sensorial representa as principais alterações responsáveis pelo aumento do risco de queda na população idosa (GALLAHUE; OZMUN; GOODWAY, 2013).

A causalidade das quedas pode ser advinda de fatores intrínsecos, em que são associadas às alterações do próprio processo de envelhecimento; de fatores extrínsecos, nos quais os riscos estão associados ao meio ambiente e atividades diárias; e por fim, de fatores comportamentais, como o uso de álcool, calçado inadequado, polifarmácia, dentre outros (PAIXÃO; HECKEMAN, 2016, p.1651).

A ocorrência de queda na população idosa chega a $30 \%$ por ano, naqueles maiores de 80 anos esse número aumenta para 40\%, e 50\% nos idosos institucionalizados. Em média, 2,5\% dos idosos vítimas de quedas necessitam ser hospitalizados e apenas metade desses sobreviverá após um ano (BRASIL, 2006).

Entre os anos de 1996 e 2012, a taxa de internação por quedas nas capitais do Brasil passou de 2,58 para 41,37 a cada 10.000 idosos. Já a proporção de mortalidade aumentou de 1,25 para 3,75 a cada 10.000 idosos (ABREU et al., 2018).

A Sociedade Brasileira de Geriatria e Gerontologia (2008), define queda como “deslocamento não intencional do corpo para um nível inferior à posição inicial com incapacidade de correção em tempo hábil, determinado por circunstâncias multifatoriais 
comprometendo a estabilidade". As quedas podem sinalizar um desequilíbrio nos sistemas osteomuscular e/ou neurológico, resultante de uma baixa capacidade funcional e indicando um declínio na saúde.

A ocorrência de quedas está entre as principais causas externas de mortalidade em pessoas idosas no Brasil. Além disso, se tornou um problema de saúde pública, com alto índice de internações e mortalidades, gerando altos custos econômicos para os serviços de saúde devido à hospitalização ou à institucionalização, além de gastos dos familiares com cuidados e medicações (PINHO et al., 2012) (ANTES et al., 2013).

Uma vez ocorrida a queda, há tendência ao imobilismo e suas intercorrências, como diminuição da capacidade respiratória, surgimento de lesões por pressão, alterações musculoesqueléticas, dentre outros, podendo culminar em óbitos (SILVA; FILONI; SUGUIMOTO, 2017).

A fisioterapia é de extrema importância no caráter preventivo desse evento, na medida em que pode reestabelecer e melhorar a capacidade funcional do idoso, proporcionando uma maior autonomia e qualidade de vida (OLIVEIRA et al., 2017).

Diante o exposto, esta pesquisa teve como objetivo descrever a mortalidade de idosos em decorrência de episódios de quedas, em Minas Gerais no período entre 2008 e 2018, especificamente buscou-se evidenciar como a fisioterapia pode ajudar a minimizar a ocorrência deste evento.

\section{MATERIAIS E MÉTODOS}

Trata-se de uma pesquisa descritiva, quantitativa, quanto ao período de segmento, tem-se um estudo longitudinal retrospectivo.

Para atender a finalidade da pesquisa, foram utilizados dados secundários do Departamento de Informática do SUS (DATASUS), especificamente do Sistema de Informação sobre Mortalidade (SIM), disponível no seguinte endereço eletrônico: www.datasus.gov.br. A escolha do banco de dados do DATASUS/SIM se baseia pela relevância de informações em saúde, pela alta confiabilidade dos dados, e por ser de rápido e fácil acesso.

O percurso metodológico da pesquisa encontra-se representado na Figura 1. 
FIGURA 1 - Fluxograma metodológico da presente pesquisa.

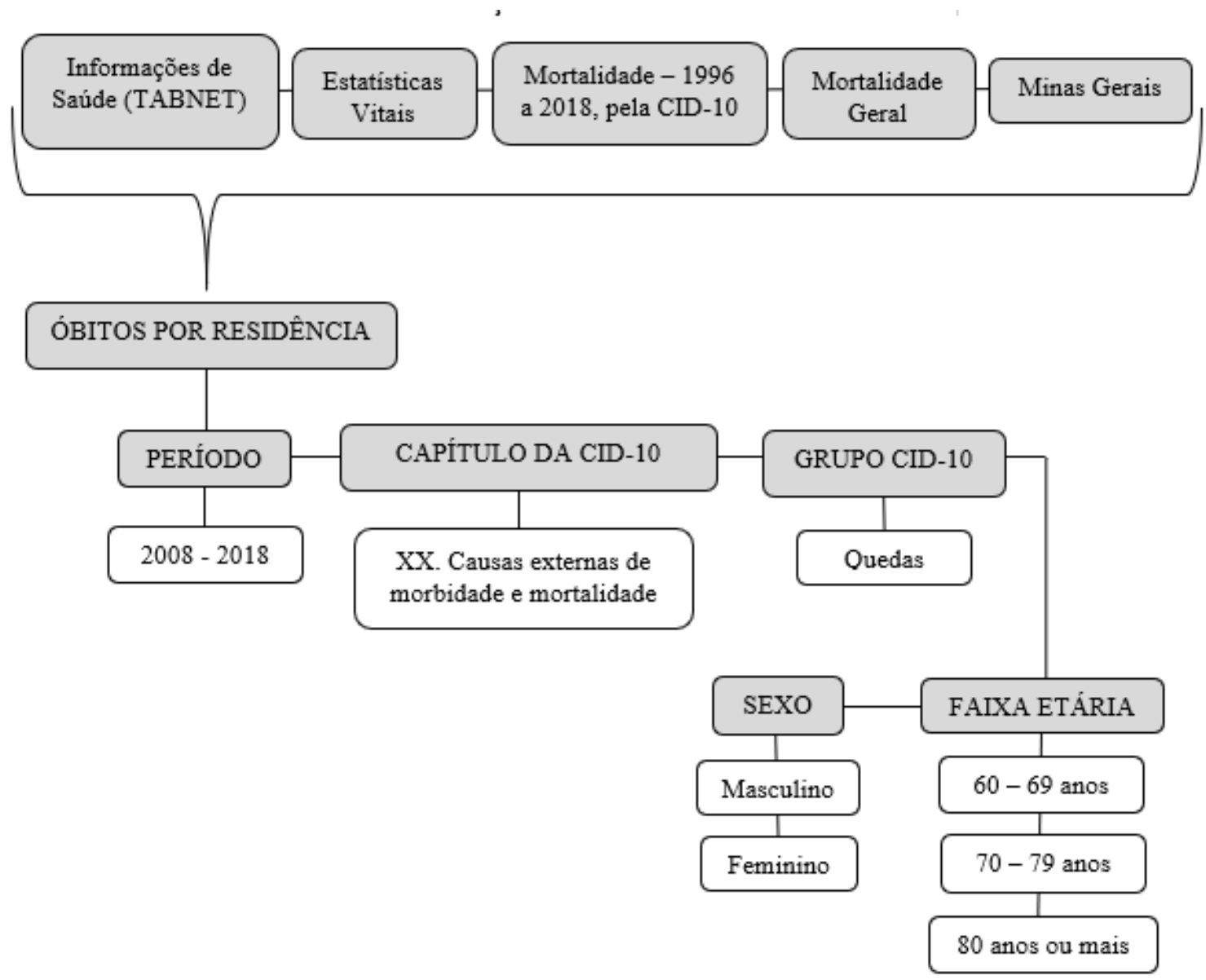

Fonte: Informações de Saúde de acordo com o DATASUS/SIM. Disponível no endereço eletrônico: www.datasus.gov.br

Os dados utilizados nesta pesquisa compreenderam o limite temporal entre 2008 e 2018. O marco cronológico foi definido por se tratar de dados atuais disponíveis nos últimos 10 anos no site do DATASUS/SIM. A faixa etária da amostra foi definida de acordo com o critério adotado no Brasil, onde são consideradas idosas aquelas pessoas com 60 anos ou mais (BRASIL, 2013). A coleta de dados foi realizada e revisada entre os dias 05 e 07 de julho de 2020.

Os dados obtidos foram digitalizados e estruturados em planilhas no software Microsoft Excel 2016, e foram analisados de acordo com a estatística básica descritiva, sendo posteriormente apresentados em forma de gráficos. 


\section{RESULTADOS E DISCUSSÃO}

No período entre 2008 e 2018, ocorreram 10.038 óbitos por quedas em idosos em Minas Gerais. Destes, 5.035 foram de mulheres (50,16\%) e 5.003 de homens (49,84\%). Alguns estudos também relacionam maior incidência em mulheres em diferentes estados do Brasil (ROSA et al., 2015) (SOARES et al., 2014) (SOUSA et al., 2016). Contraditoriamente, outros estudos apontam maior incidência em homens (MACIEL et al., 2010) (GOMES; BARBOSA; CALDEIRA, 2010) (ANDRADE et al., 2017).

Tratando a queda como um fenômeno que ocorre em ambos sexos, a fisioterapia apresenta intervenções eficientes para a prevenção desse evento. Vários estudos mostram a eficiência de um programa fisioterapêutico de caráter preventivo relacionado às quedas, nos quais são apresentados variados métodos e consequentes melhorias significativas do equilíbrio, força muscular, amplitude de movimento, marcha e funcionalidade; e, dessa forma, obtém-se a diminuição do risco de quedas (ZAPPE; FILIPPIN, 2016) (OLIVEIRA et al., 2017) (SILVA et al., 2019).

De acordo com o Gráfico 1, o ano com maior mortalidade por quedas em idosos, de ambos os sexos, foi 2018, em que foram registradas 1.404 mortes, sendo 694 de homens e 710 de mulheres. Já o ano de 2008 registrou a menor mortalidade, 280 óbitos de homens e 270 de mulheres. Nota-se que o ano de 2018 apresentou índice de mortalidade 155,27\% maior que o ano de 2008. 
Gráfico 1: Óbitos por quedas entre o sexo masculino e feminino, no período entre 2008 - 2018, em Minas Gerais.

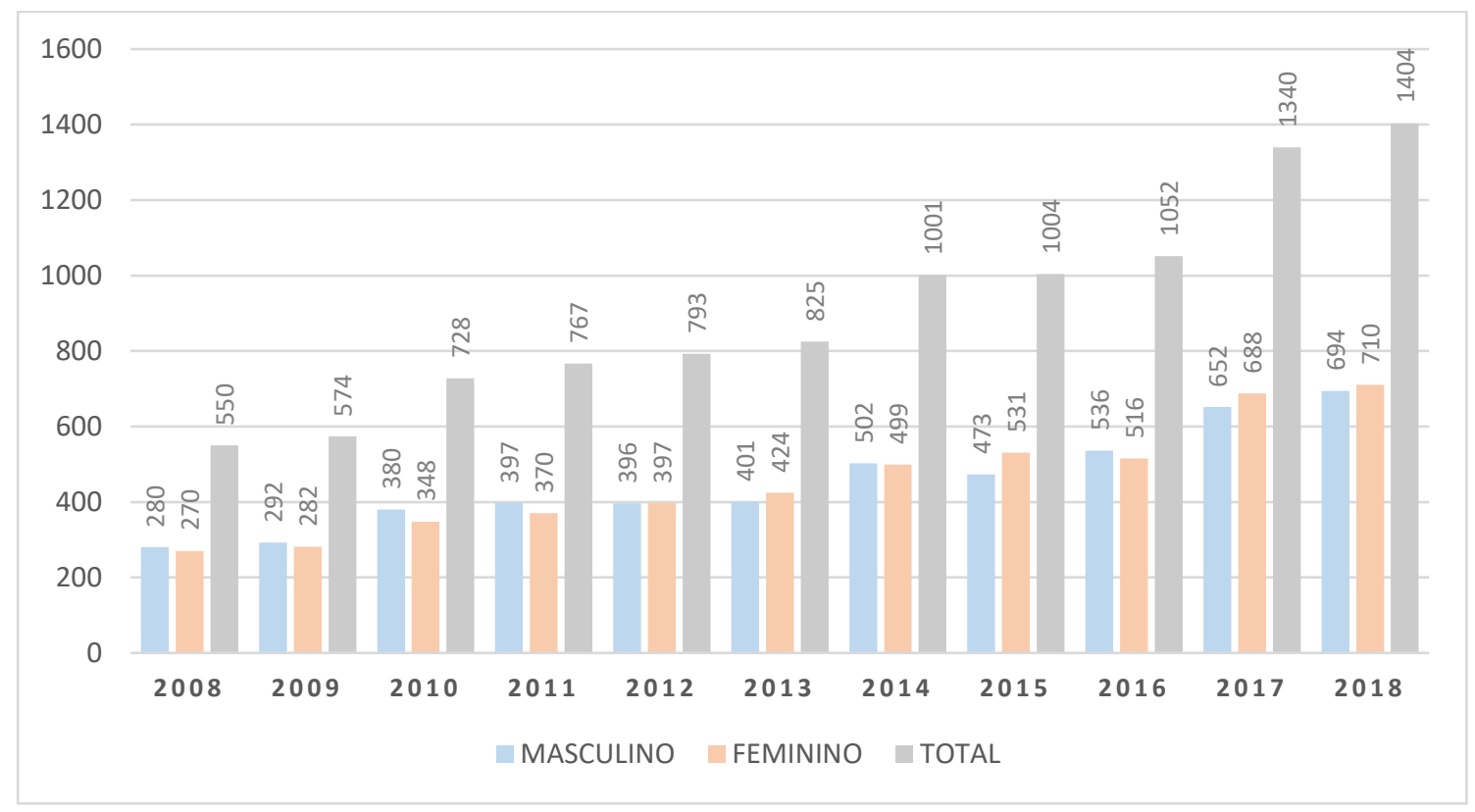

Fonte: Elaborado pelos autores.

O estudo de Gomes, Barbosa, Caldeira (2010) relacionou o aumento de óbitos por quedas em idosos em Minas Gerais nos anos 2000-2007. No ano 2000 havia registros de 14,3/100.000 homens e 9,4/100.000 mulheres e, em 2007, os dados evoluíram para 27,4/100.000 homens e 19,1/100.000 mulheres. Esse resultado correlaciona-se ao presente estudo, demonstrando que o índice de mortalidade aumenta a cada ano, e que são necessárias ações preventivas para reduzir essas estatísticas.

A reabilitação do idoso pode mudar essa realidade a partir de intervenções como orientações, organização domiciliar, exercícios terapêuticos, fortalecimento muscular, treino de equilíbrio, de marcha e treino funcional, além da atuação na minimização das consequências do imobilismo (PAIXÃO; HECKEMAN, 2016).

Sabendo-se que alteração de equilíbrio e agilidade são fatores predisponentes de quedas, o estudo de Franciulli et al. (2015) teve como objetivo, demonstrar a efetividade do atendimento fisioterapêutico em idosos com histórico de quedas. Os indivíduos foram separados em dois grupos: 8 idosos no G1 (hidroterapia) e 6 idosos no G2 (cinesioterapia), foram avaliados o equilíbrio, atividade de vida diária e agilidade. Como resultado, os autores não identificaram superioridade de uma intervenção ao compará-la com a outra, mas observaram melhoras 
significativas em todos os aspectos avaliados, e por consequência, a diminuição do risco de quedas.

Analisando a prevalência de mortalidade por período e sexo, (Gráfico 1), entre 2008 e 2011, houve uma prevalência de óbitos no sexo masculino. Em 2012 e 2013, a maior mortalidade ocorreu no sexo feminino. Em 2014 os óbitos prevaleceram entre os homens e em 2015 em mulheres. Já em 2016, o número de mortes de homens foi superior e nos anos de 2017 e 2018, as mulheres voltaram a representar maior taxa de mortalidade. No que diz respeito a relação de óbitos e sexo, não foram encontrados estudos com diferença significativa entre mortalidade por quedas em idosos do sexo masculino e feminino entre os períodos analisados.

Considerando a relevância da queda no cotidiano da pessoa idosa, os dados mostrados evidenciam a importância do trabalho preventivo da Fisioterapia. O fisioterapeuta inserido na Atenção Primária da Saúde teria como alternativa trabalhar ações de educação voltadas à prevenção de quedas, envolvendo idosos homens e mulheres.

Como pode ser observado no Gráfico 2, a faixa etária em que ocorreu maior número de óbitos foi 80 anos ou mais, que correspondeu a 57,18\% do total de casos; $25,69 \%$ dos óbitos ocorreram na faixa etária 70 a 79 anos e 17,12\% entre 60 e 69 anos.

Antes, Schneider, d'Orsi (2015) analisaram a mortalidade por quedas em idosos nos anos de 1999-2008, no Brasil e no estado de Santa Catarina. Foi observado desfecho similar, tendo em vista os anos que antecedem o período do presente estudo. Nos resultados, o número de óbitos na faixa etária de 80 anos ou mais é maior quando comparado ao grupo etário de 70-79 anos e de 60-69 anos. Os autores analisaram que a faixa etária maior que 80 anos obteve superioridade em todo o período estudado, e que houve um acréscimo no índice de mortalidade de 7,2\% no Brasil e 13,6\% em Santa Catarina.

Em uma revisão integrativa, Wingerter et al. (2020) investigaram oito estudos publicados entre os anos de 2007-2016, todos os estudos apresentaram a relação de maior risco de óbitos em idosos com mais idade. 
Gráfico 2: Óbitos por quedas considerando a faixa etária e sexo, no período de 2008 a 2018, em Minas Gerais

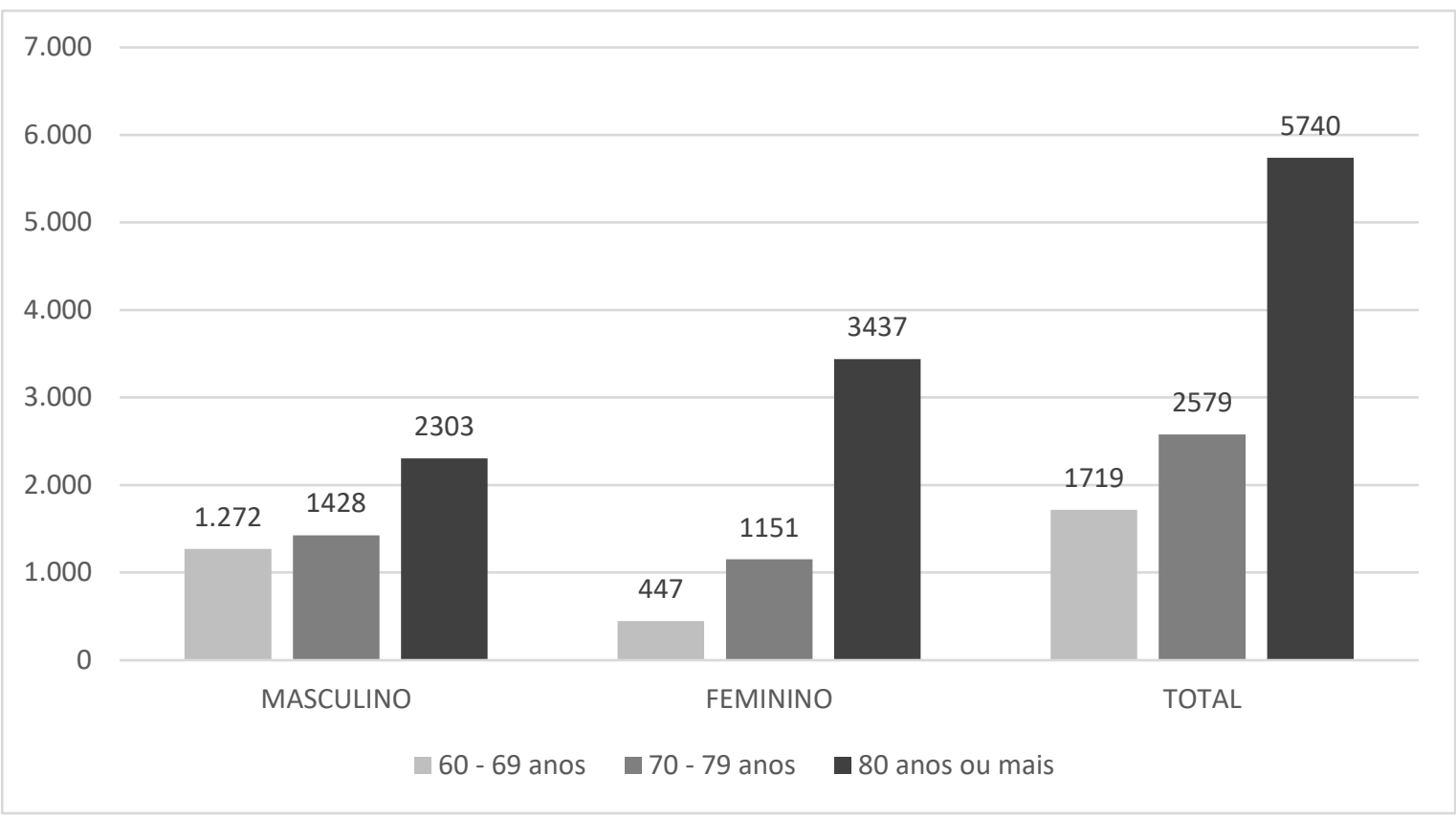

Fonte: Elaborado a partir de dados disponibilizados pelo DATASUS/SIM.

Tomando como base essa estatística verifica-se que a atuação do fisioterapeuta é imprescindível na prevenção de quedas nas diferentes faixas etárias das pessoas idosas; por meio de ações educativas, exercícios físicos orientados e regulares, terapia comportamental é possível manter o idoso ativo, consequentemente preservando sua capacidade funcional.

O predomínio de mortes dos idosos maiores de 80 anos é justificável pelo fato desse grupo de pessoas ser mais passível as alterações do envelhecimento, uma vez que apresentam maior vulnerabilidade e consequentemente maior declínio funcional, o que o torna mais susceptível às complicações decorrentes da queda, que podem culminar em óbito (SIQUEIRA et al., 2007). Tal realidade, aponta a necessidade de um trabalho de reabilitação para prevenção de quedas em octogenários.

Conforme o Gráfico 2, nas faixas etárias de 60 a 69 e de 70 a 79 anos a mortalidade foi mais prevalente entre os homens (1.272 e 1.428, respectivamente); contrariamente, em pessoas com 80 anos ou mais, predominaram os óbitos em mulheres (3.437). Nesse aspecto, o estudo de Rosa et al. (2015) apresentou resultados semelhantes, observou-se prevalência do sexo masculino entre o grupo etário 60-69 anos e 70-79 anos, já nos idosos com 80 anos ou mais, as mulheres apresentaram maior índice de óbitos por quedas.

De acordo com Gawryszewski et al. (2004), no ano de 2000, ocorreram 2.030 óbitos por 
quedas em idosos, e as quedas constituíram a terceira causa de mortalidade por causas externas no Brasil; em se tratando de morbidade, as quedas ocuparam o primeiro lugar, com 49.980 $(56,1 \%)$ casos de hospitalização. Em um estudo recente, Vey et al. (2016) confirmaram a crescente taxa de hospitalização e óbitos a cada ano no Brasil; no ano de 2009, ocorreram 69.880 internações e 6.243 óbitos; em 2010, foram registrados 81.293 hospitalizações e 7.427 mortes; e no ano de 2011, os número foram ainda maiores, 85.945 internações e 7.722 óbitos.

Apesar do aumento gradual de hospitalizações, óbitos e intercorrências oriundas das quedas, a fisioterapia pode ajudar a reduzir esses números, a partir de sua participação indispensável na identificação dos fatores de riscos, na promoção de saúde, na prevenção e reabilitação do idoso (AVEIRO et al., 2011).

O medo de cair novamente é uma das principais consequências da queda e pode acarretar a restrição da vítima ao leito, ocasionada pela perda da confiança ao deambular. Essa ocorrência diminui a participação social do idoso, além de predispor à ansiedade, depressão e declínio funcional, tornando o idoso mais susceptível a novos episódios de quedas (LOPES et al., 2009).

A fisioterapia pode atuar tanto na prevenção quanto na reabilitação do imobilismo. Quando o indivíduo está submetido ao imobilismo, a fisioterapia possui abordagens essenciais para melhora da flexibilidade, força muscular, atividade de vida diária, marcha, além da reaquisição da funcionalidade e independência do idoso (MACEDO; GAZZOLA; NAJAS, 2008).

Com base nos resultados apresentados e visto que a ocorrência de queda é um problema de saúde pública, tornam-se imprescindíveis ações de saúde voltadas para compreender o retrato da população idosa e a prevenção da ocorrência desse evento indesejável. A fisioterapia se mostra indispensável nesse contexto, principalmente na detecção preventiva dos fatores de risco, uma vez que o atendimento é realizado de forma holística, consoante ao modelo biopsicossocial, avaliando não só o paciente, mas todo o meio em que ele vive (SCHNEIDER, 2010).

\section{CONCLUSÃO}

Ao considerar o sexo, observa-se que a mortalidade variou muito no período estudado não sendo possível estabelecer uma relação direta entre óbito por quedas e sexo. Ao analisar a 
idade, nota-se que a mortalidade entre os idosos com 60-69 anos e 70-79 anos é prevalente em homens, e as mulheres predominam a faixa etária com 80 anos ou mais.

É notório que a mortalidade por quedas nesta população vem aumentando a cada ano no estado de Minas Gerais, e fica evidente a imperiosa necessidade de implantação de ações governamentais de prevenção e educação, voltadas para combater esse evento indesejável.

O fisioterapeuta é um profissional de suma importância nas ações de saúde, tais como, promoção, prevenção, reabilitação e educação, e no cenário da mortalidade e morbidade por quedas, é fundamental a sua participação para mudar tais estatísticas.

Pode-se concluir que apesar da mortalidade por quedas atingir todos os grupos etários dos idosos, o risco aumenta ainda mais a partir de 80 anos, o que requer atenção especial a esse grupo, uma vez que as intercorrências são mais frequentes e agressivas. É fundamental uma abordagem da fisioterapia nesse contexto, para ajudar a reduzir as intercorrências das quedas, inclusive a mortalidade.

\section{REFERÊNCIAS}

ABREU, D. R. O. M.; NOVAES, E. S.; OLIVEIRA, R. R.; MATHIAS, T. A. F.; MARCON, S. S. Internação e mortalidade por quedas em idosos no Brasil: análise de tendência. Ciênc. Saúde Coletiva. v.23, n4. Rio de Janeiro, 2018. Disponível em: https://www.scielo.br/scielo.php?script=sci_arttext\&pid=S141381232018000401131\#: :text=A\%20taxa\%20de\%20mortalidade\%20de,ano\%2C\%20entre\%2 01996\%20e\%202012. DOI: https://doi.org/10.1590/1413-81232018234.09962016. Acesso em: 06 ago. 2020

ANDRADE, I. R.; SOUZA, E. A.; LUZ, L. A.; JUNIOR, E. P. P. Características e gastos com hospitalização por quedas em idosos na Bahia. J. Health Sci. Inst. Bahia, 2017. v.35, n.1. Disponível em: https://www.unip.br/presencial/comunicacao/publicacoes/ics/edicoes/2017/01_janmar/V35_n1_2017_28a31.pdf. Acesso em: 04 ago.2020

ANTES, D. L.; SCHNEIDER, I. J. C.; BENEDETTI, T. R. B.; D’ORSI, E. Medo de queda recorrente e fatores associados em idosos de Florianópolis, Santa Catarina, Brasil. Rev. Saúde Pública. Rio de Janeiro, 2013. v.29, n.4. Disponível em: https://www.scielo.br/pdf/csp/v29n4/13.pdf. DOI: https://doi.org/10.1590/S0102311X2013000400013. Acesso em: 04 ago.2020

ANTES, D. L.; SCHNEIDER, I. J. C.; D’ORSI, E. Mortalidade por quedas em idosos: estudo de série temporal. Rev. Bras. Gerontol, v.18, n.4, p.769-778. Rio de Janeiro, 2015. 
Disponível em: https://www.scielo.br/pdf/rbgg/v18n4/pt_1809-9823-rbgg-18-04-00769.pdf. DOI: http://dx.doi.org/10.1590/1809-9823.2015.14202. Acesso em: 08 ago. 2020.

AVEIRO, M. C.; ACIOLE, G. G.; DRIUSSO, P.; OISCHI, J. Perspectiva da participação do fisioterapeuta no Programa Saúde da Família na atenção à saúde do idoso. Ciênc. Saúde Coletiva, v.16, n.1, p.1467-1478. Rio de Janeiro, 2011. Acesso em: https://www.scielo.br/scielo.php?script=sci_arttext\&pid=S1413-81232011000700082. DOI: https://doi.org/10.1590/S1413-81232011000700082. Acesso em: 08 ago. 2020.

BRASIL. Lei n. 8.842, de 4 de janeiro de 1994. Dispõe sobre a Política Nacional do Idoso, cria o Conselho Nacional do Idoso e dá outras providências. Brasília; 1994. Disponível em: http://www.planalto.gov.br/ccivil_03/Leis/L8842.htm. Acesso em: 05 ago. 2020

BRASIL, Ministério da Saúde. Cadernos de Atenção Básica. Envelhecimento e Saúde da Pessoa Idosa. Brasília-DF, 2006. Disponível em:

http://bvs.saude.gov.br/bvs/publicacoes/evelhecimento_saude_pessoa_idosa.pdf. Acesso em: 06 ago. 2020

BRASIL, Ministério da Saúde. Estatuto do Idoso. 3.ed, Brasília-DF, 2013. Disponível em: https://bvsms.saude.gov.br/bvs/publicacoes/estatuto_idoso_3edicao.pdf. Acesso em: 06 ago. 2020

FRANCIULLI, P. M.; SOUZA, G. B.; ALBIACH, J. F.; SANTOS, K. C. P.; BARROS, L. O.; TEOTÔNIO, N. S.; ANDRADE, F. S.; BIGONGIARI, A.; BARBANERA, M.

Efetividade da hidroterapia e da cinesioterapia na reabilitação de idosos com histórico de quedas. Estud. Interdiscip. Envelhec, v.20, n.3, p.671-686. Porto Alegre, 2015. Disponível em: https://pesquisa.bvsalud.org/portal/resource/pt/biblio-868923. Acesso em: 08 ago. 2020.

GALlAHUE, D. L.; OZMUN, J. C.; GOODWAY, J. D. Compreendendo o Desenvolvimento Motor: bebês, crianças, adolescentes e adultos. 7ed. Porto Alegre: Artmed, 2013. p.416-417.

GOMES, L. M. X.; BARBOSA, T. L. A.; CALDEIRA, A. P. Mortalidade por causas externas em idosos em Minas Gerais, Brasil. Esc. Anna Nery, v.14, n.1, Rio de Janeiro, 2010. DOI: https://doi.org/10.1590/S1414-81452010000400018. Disponível em:

https://www.scielo.br/scielo.php?script=sci_arttext\&pid=S1414-81452010000400018. DOI: Acesso em: 07 ago.2020

IBGE - Instituto Brasileiro de Geografia e Estatística. Mudança Demográfica no Brasil no Início do Século XXI. Subsídios para as projeções da população. Rio de Janeiro, 2015. Estudos e Análises, n³, p. 146. Disponível em: https://biblioteca.ibge.gov.br/visualizacao/livros/liv93322.pdf. Acesso em: 04 ago. 2020.

IBGE - Instituto Brasileiro de Geografia e Estatística. Projeção da População. Brasil e das Unidades da Federação. Rio de Janeiro, 2018. Disponível em:

https://biblioteca.ibge.gov.br/visualizacao/livros/liv101597.pdf. Acesso em: 04 ago. 2020. 
LOPES, K. T.; COSTA, D. F.; SANTOS, L. F.; CASTRO, D. P.; BASTONE, A. C. Prevalência do medo de cair em uma população de idosos da comunidade e sua correlação com mobilidade , equilíbrio dinâmico, risco e histórico de quedas. Rev. Bras Fisioter, v.13, n. 3, p.223-229. São Carlos - SP, 2009. Disponível em: https://www.scielo.br/scielo.php?script=sci_arttext\&pid=S1413-35552009000300006. DOI: https://doi.org/10.1590/S1413-35552009005000026. Acesso em: 10 ago. 2020.

MACEDO, C.; GAZZOLA, J. M.; NAJAS, M. Síndrome da fragilidade no idoso: importância da fisioterapia. Arquivos Brasileiros de Ciência da Saúde, n.33, n.3, p.177-184. São Paulo, 2008. Disponível em: http://files.bvs.br/upload/S/1983-2451/2008/v33n3/a177-184.pdf.

Acesso em: 07 ago. 2020.

MACIEL, S. S. S. V.; VIEIRA, W. M. TEOTÔNIO, P. M.; BARBOSA, G. G.; LIMA, V. G. C.; OLIVEIRA, T. F.; SILVA, E. T. C. Perfil epidemiológico das quedas em idosos residentes em capitais brasileiras utilizando o Sistema de Informação sobre Mortalidade. Rev.

AMRIGS, v.54, n.1, p. 25-31. Maceió, 2010. Disponível em:

https://pesquisa.bvsalud.org/portal/resource/pt/lil-685592. Acesso em: 02 ago. 2020

OLIVEIRA, H. M. L.; RODRIGUES, L. F.; CARUSO, M. F. B.; FREIRE, N. S. A. Fisioterapia na prevenção de quedas em idosos: revisão de literatura. Revista

Interdisciplinar de Estudos Experimentais, v.9, n.1, p.43-47, 2017. Disponível em: http://docs.bvsalud.org/biblioref/2018/11/964842/2898-8949-1-sm.pdf. Acesso em: 04 ago. 2020.

PAIXÃO, C. M. J.; HECKMAN, M. F de. Distúrbios de Postura, Marcha e Quedas. In: FREITAS, E. V. de; PY, L. Tratado de Geriatria e Gerontologia. 4. ed. Rio de Janeiro: Guanabara Koogon, 2016. p. 1642-1657.

PINHO, T. A. M.; SILVA, A. O.; TURA, L. F. R.; MOREIRA, M. A. S. P.; GURGEL, S. N.; SMITH, A. A. F.; BEZERRA, V. P. Avaliação do risco de quedas em idosos atendidos em unidade básica de saúde. Rev. esc. enferm. USP, v. 46, n. 2, p. 320-327, 2012. Disponível em: https://www.scielo.br/scielo.php?script=sci_arttext\&pid=S0080-62342012000200008. DOI: http://dx.doi.org/10.1590/S0080-62342012000200008. Acesso em: 04 ago.2020.

ROSA, T. S. M.; MORAES, A. B.; PERIPOLLI, A.; SANTOS, V. A. V. F. Perfil epidemiológico de idosos que foram a óbito por queda no Rio Grande do Sul. Rev. Bras. Geriatr. Gerontol. v.18, n.1, p.59-69. Disponível em: https://www.scielo.br/pdf/rbgg/v18n1/1809-9823-rbgg-18-01-00059.pdf. DOI: https://doi.org/10.1590/1809-9823.2015.14017. Acesso em: 07 ago. 2020.

SCHNEIDER, A. R. S. Envelhecimento e quedas: a fisioterapia na promoção e atenção à saúde do idoso. RBCEH, v.7, n.2, p.293-303. Passo Fundo, 2010. Disponível em: http://seer.upf.br/index.php/rbceh/article/view/414/pdf. DOI: https://doi.org/10.5335/rbceh.2012.414. Acesso em: 07 ago. 2020

SILVA, J. L.; FILONI, E.; SUGUIMOTO, C. M. Análise do incremento da força muscular para reaquisição de ortostatismo em idosos com síndrome do imobilismo temporário. Acta 
Fisiatr. v.24, n3, p.113-119. São Paulo, 2017. DOI: https://doi.org/10.5935/01047795.20170021. Disponível em: https://www.revistas.usp.br/actafisiatrica/article/view/153686/150110. Acesso em: 04 ago.2020.

SILVA, L. P.; DUARTE, M. P. S.; SOUZA, C. C. B.; LINS, C. C. S. A.; CORIOLANO, M. G. W. S.; GOMES, O. G. Efeitos da prática mental associada a fisioterapia motora sobre a marcha e o risco de quedas na doença de Parkinson: estudo piloto. Fisioter. Pesqui. v.26, n. 2. São Paulo, 2019. Disponível em: https://www.scielo.br/scielo.php?script=sci_arttext\&pid=S1809-29502019000200112. DOI: https://doi.org/10.1590/1809-2950/17012926022019

. Acesso em: 07 ago. 2020

SIQUEIRA, F. V.; FACCHINI, L. A.; PICCINI, R. X.; TOMASI, E.; THUMÉ, E.; SILVEIRA, D. S.; VIEIRA, V.; HALLAL, P. C. Prevalência de quedas em idosos e fatores associados. Rev. Saúde Pública. v.41, n.5, p.749-756. Disponível em: https://www.scielo.br/pdf/rsp/v41n5/6188.pdf. Acesso em: 04 ago. 2020.

SOARES, W. J. S.; MORAES, S. A.; FERRIOLLI, E.; PERRACINI, M. R. Fatores associados a quedas e quedas recorrentes em idosos: estudo de base populacional. Rev. Bras. Geriatr. Gerontol. v.17, n.1, p. 49-60, Rio de Janeiro, 2014. Disponível em: https://www.scielo.br/pdf/rbgg/v17n1/1809-9823-rbgg-17-01-00049.pdf. Acesso em: 07 ago. 2020.

SOCIEDADE BRASILEIRA DE GERIATRIA E GERONTOLOGIA. Quedas em idosos: prevenção Projeto Diretrizes - Associação Médica Brasileira e Conselho Federal de Medicina. SBGG, Rio de Janeiro, 26 out. 2008. Disponível em: https://sbgg.org.br/wpcontent/uploads/2014/10/queda-idosos.pdf. Acesso em: 05 ago. 2020.

SOUSA, R. A.; BERNADES, D. C. F.; SANTANA, J. A.; BARREIRA, L. M. Prevalência de quedas em idosos na comunidade. Rev. Saúde e Pesquisa, v.9, n.3, p. 547-555. Goiás, 2016. Disponível em:

https://periodicos.unicesumar.edu.br/index.php/saudpesq/article/view/5605/2922. DOI: http://orcid.org/0000-0002-0617-8112. Acesso em: 07 ago.2020.

VEY, A. P. Z.; DALENOGARE, J. F.; SILVA, A. C.; MARQUES, C. M. G. Quedas e frequência de internação e mortalidade em idosos o Brasil e no Rio Grande do Sul.

Fisioterapia Brasil, v.17, n.6, p.559-565. Rio Grande do Sul, 2016. Disponível em: https://portalatlanticaeditora.com.br/index.php/fisioterapiabrasil/article/view/697/1515. Acesso em: 08 ago. 2020.

WINGERTER, D. G.; BARBOSA, I. R.; MOURA, L. K. B.; MACIEL, R. F.; ALVES, M. S. C. F. Mortalidade por queda em idoso: uma revisão integrativa. Rev. Ciência Plural, v.6, n.1, p.119-136. Rio Grande do Norte, 2020. Disponível em:

https://periodicos.ufrn.br/rcp/article/view/18366/12532. Acesso em: 07 ago. 2020. 
ZAPPE, A. R. S.; FILIPPIN, N. T. Intervenção fisioterapêutica no equilíbrio postural de idosos. Fisioterapia Brasil, v.17, n.5, p.505-516. Rio Grande do Sul, 2016. Disponível em: http://docs.bvsalud.org/biblioref/2018/05/883355/intervencao-fisioterapeutica-no-equilibriopostural-de-idosos.pdf. Acesso em: 07 ago. 2020.

\section{Declaração de Interesse}

Os autores declaram não haver nenhum conflito de interesse

\section{Financiamento}

Financiamento próprio

\section{Colaboração entre autores}

Castro concebeu o estudo, participou do planejamento da pesquisa, realizou a coleta e análise dos dados, criação de tabelas e figuras, bem como escreveu o manuscrito.

Filho participou do planejamento do estudo, da revisão da redação da pesquisa.

Sousa colaborou com a revisão do texto e adição de partes significativas.

Silva participou do planejamento do estudo, orientou todas as etapas da pesquisa, contribuiu com o desenho $e$ interpretação dos dados, bem como a revisão do texto e adição de partes significativas. 